\title{
Update on Current Treatments for Migraine
}

\author{
An Expert Interview with Stewart J Tepper
}

Geisel School of Medicine at Dartmouth, Hanover, NH, US

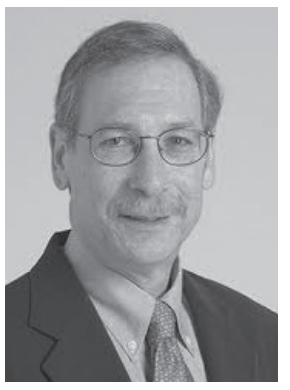

DOI: https://doi.org/10.17925/USN.2018.14.2.71

\section{Stewart J Tepper}

Stewart J Tepper, MD, FAHS, is Professor of Neurology at Geisel School of Medicine at Dartmouth (Dartmouth Medical School), Hanover, NH, and Director, Dartmouth Headache Clinic, Department of Neurology, Dartmouth-Hitchcock Medical Center, Lebanon, NH, US. He lectures nationally and internationally on all aspects of headache medicine. His interests are in patient care and education, with research in the causes of headaches, their treatments, and patient outcomes. He has published over 375 peer-reviewed papers on headache topics and eight books. Professor Tepper serves on the Board of Directors and Education Committees of the American Headache Society (AHS) and belongs to multiple societies including the American Academy of Neurology. He is Director of the AHS Scottsdale Headache symposium and Editor-in-Chief of the journal Headache Currents.

\section{Keywords}

Migraine, calcitonin gene-related peptide, monoclonal antibody, gepants

Disclosure: Stewart J Tepper has received grants or research support from Alder BioPharmaceuticals, Allergan Amgen, ATI, Dr Reddy's, ElectroCore, eNeura, Teva, and Zosano; consulted and/or served as advisor to Acorda, Alder BioPharmaceuticals, Alexsa, Allergan, Amgen, ATI, Axsome, Charleston Laboratories, DeepBench, Dr. Reddy's, ElectroCore, Eli Lilly, eNeura, the Gerson Lehman Group, Guidepoint Global, Neurolief, Novartis, Pernix, Scion Neurostim, Slingshot Insights, Teva, and Zosano; holds stock options in ATI; has received royalties from Springer; and receives salary from Dartmouth-Hitchcock Medical Center and the American Headache Society. $\mathrm{He}$ is also a member of the journal's editorial board.

Review Process: This is an expert interview and, as such, has not undergone the journal's standard peer review process.

Acknowledgment: Medical writing assistance was provided by Ray Ashton of Touch Medical Media and was supported by Touch Medical Media.

Authorship: The named author meets the Internationa Committee of Medical Journal Editors (ICMJE) criteria for authorship of this manuscript, takes responsibility for the integrity of the work as a whole, and has given final approval for the version to be published.

open Access: This article is published under the Creative commons Attribution Noncommercial License, which permits any noncommercial use, distribution, adaptation, and reproduction provided the original author and source are given appropriate credit. (c) The Author 2018.

Received: September 26, 2018

Published Online: November 6, 2018

Citation: US Neurology. 2018;14(2):71-2

Corresponding Author: Stewart J Tepper Department of Neurology, Dartmouth-Hitchcock Medical Center, One Medical Center Drive, Lebanon, $\mathrm{NH}$ 03756-1000, US. E: Stewart.J.Tepper@Dartmouth.edu

Support: No funding was received in

the publication of this article. $n$ the last year, a number of exciting new treatments for migraine have been approved by regulatory authorities. In particular, several monoclonal antibodies have revolutionized every aspect of treatment. In an expert interview, Professor Stewart J Tepper describes these new treatments and speculates on their impact on the management of migraine.

\section{Q. What important developments in the understanding of the pathophysiology of migraine have guided research into new therapeutic targets, such as CGRP?}

There is overwhelming evidence that calcitonin gene-related peptide (CGRP) is a target for migraine treatments. For example, levels are elevated during migraine, but are lower before and after an attack, and CGRP receptors are found in important locations in the nervous system implicated in migraine pathogenesis. Therapies targeting CGRP receptors are effective in migraine. When CGRP is released in the meninges, it causes vasodilation and inflammation, so antagonism has the potential for a number of therapeutic effects.

Another potential target is pituitary adenylate cyclase activating polypeptide-38 (PACAP-38). When this peptide is infused, it is associated with initiating a migraine in a delayed manner. An anti PACAP-38 receptor (anti-PACAP-1) monoclonal antibody (MAb) is in development, with results from a phase II trial expected in 2019 (NCT03238781).

The rationale for discovering a new target is to identify possible targets in brain locations that appear to be involved with migraines and prove the link. It is likely that more targets will be identified in the future.

\section{Q. In the case of CGRP, how has the discovery of this target translated into the development of new anti-CGRP treatments?}

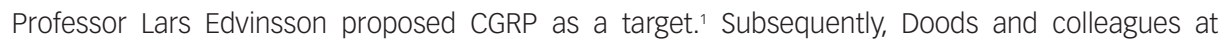
Boehringer Ingelheim synthesised part of the CGRP receptor and then developed a series of anti-CGRP small protein molecules that could bind and antagonise it. These small molecules blocked the CGRP receptor, preventing vasodilation but not causing vasoconstriction. The first compound tested in humans was BIBN 4096 BS, now called olcegepant, a small molecule CGRP-receptor antagonist with high affinity and specificity for the human CGRP receptor. ${ }^{2}$ Intravenous administration was effective in treating acute attacks of migraine with few side effects. 
Subsequently, many pharmaceutical companies developed these small molecule CGRP receptor blockers which are known as gepants, and six compounds have shown efficacy for the acute treatment of episodic migraine. However, liver toxicity occurred with three of these. Telcagepant was associated with severe liver symptoms, and development was stopped. The gepants generally have excellent tolerability, and several should soon be submitted for regulatory approval, including ubrogepant and rimegepant for acute migraine treatment, and which have not shown significant liver toxicity signals. Daily administration of atogepant was recently shown to prevent episodic migraine. Currently, gepants are being evaluated for both acute and preventative treatment of migraines.

\section{Q. What is the current status of monoclonal antibodies for the treatment of migraine?}

As gepants may be associated with liver toxicity, MAbs, which are eliminated by the reticuloendothelial system, are an attractive alternative. To date, three anti-CGRP or MAbs have been approved by the US Food and Drug Administration for the prevention of migraine. The first was erenumab-aooe, approved in May 2018, and is the only MAb targeting the CGRP receptor. The other two, fremanezumab-vfrm and galcanezumab-gnlm, were approved in September 2018 and target the CGRP ligand itself. A fourth, eptinezumab, also targeting the CGRP peptide, will likely be submitted for regulatory approval in the next year. Erenumab, fremanezumab and galcanezumab are administered subcutaneously, and eptinuzumab intravenously.

\section{Q. What other promising anti-CGRP treatments are in development for the prevention of migraine?}

currently two gepants (one effective in phase II trial) are being developed for preventative migraine treatment. Three anti-CGRP MAbs are now approved and available with the fourth in development with likely submission for approval in the next year.

\section{Q. What are the potential benefits and limitations of the different classes of CGRP-directed treatments?}

It is important to emphasize the game-changing aspects of the anti-CGRP MAbs, which represent a very dramatic shift in the treatment of migraine. When erenumab became available, reportedly there were one million hits recorded on the website. The main reason for this explosion of interest and treatment was that the existing available oral medications for migraine prevention had all been developed for other indications (e.g., antidepressants, anti-epilepsy drugs and anti-hypertensives). They provide modest benefit, require titration to an effective dose, often take up to 3 months after titration for efficacy, and frequently have significant side effects. It has been estimated that $81-83 \%$ of patients stop taking these drugs over a year.

In contrast, the anti-CGRP MAbs were designed for the migraine indication, are administered monthly or quarterly rather than daily, and have minimal side effects (e.g., minor respiratory symptoms). In addition, unlike the existing therapies, in the randomized controlled trials all separated from placebo in effect within one week, all showed meaningful clinical benefit within one month, and eptinezumab demonstrated benefit within 24 hours. Patients are likely to be quite adherent with this treatment. Up to one third of patients treated with erenumab, fremanezumab, or galcanezumab experience a $75 \%$ reduction in monthly migraine days by 12 weeks, and $41-54 \%$ show this effect at 1 year with erenumab and eptinezumab. These MAbs are effective in episodic and chronic migraines, and in migraines with and without aura. Galcanezumab is also effective in preventing episodic cluster headaches, ${ }^{3}$ and studies are ongoing with fremanezumab for this indication. Their annual cost in the US in academic centers can be one-third the cost of 1 year of botulinum toxin cycles for chronic migraine.

\section{Q. Do the potential preventative therapies also have a role in the treatment of acute migraine?}

Acute migraine is an incorrect term. It is important to distinguish between episodic migraine (attacks occurring on less than 15 days/month) and chronic migraine (attacks for 15 or more days/month). Acute treatment, which can be administered for both episodic and chronic migraine days, aims to terminate that particular migraine on an as-needed basis. Acute treatment for migraine attacks and preventative treatment of migraine have long been considered different. The MAbs are not designed for acute treatment, but gepants can be used for both indications, which represents another shift in the migraine treatment paradigm.

\section{Q. How do you see anti-CGRP therapies fitting into the future landscape of acute and preventative migraine treatments?}

It is uncertain if gepants have a sustained benefit, as to date there is insufficient experience prescribing them. They have a role in patients who cannot take triptans due to vascular disease. As preventive therapy, gepants have the advantage of oral administration, and have a relatively short half-life, so are removed from the body in a few days, unlike MAbs with a half-life of 1 month. This may be important in the situation of unplanned pregnancy.

Further expert insight from Stewart J Tepper in terms of a recent full history and review of anti-CGRP therapies for the acute and preventative treatment of primary headache disorders is available. ${ }^{4} \square$

1. Edvinsson L. The journey to establish CGRP as a migraine target: a retrospective view. Headache. 2015;55:1249-55.

2. Olesen J, Diener H-C, Husstedt IW, et al. Calcitonin gene-related peptide receptor antagonist BIBN 4096 BS for the acute treatment of migraine. N Eng/ J Med. 2004;350:1104-10.

Stauffer VL, Dodick DW, Zhang Q et al. Evaluation of galcanezumab for the prevention of episodic migraine: the EVOLVE-1 randomized clinical trial. JAMA Neurol. 2018;75:1080-88.

Tepper SJ. History and review of anti-calcitonin gene-related peptide (CGRP) therapies: from translational research to treatment. Headache. 2018. DOI: 10.1111/head.13379 [Epub ahead of print]. 